\title{
Critical evaluation of the NR-1000F Auto Refractometer
}

\author{
SUPRIYO GHOSE, BARUN KUMAR NAYAK, AND JAI PAL SINGH \\ From the Dr Rajendra Prasad Centre for Ophthalmic Sciences, All India Institute of Medical Sciences, Ansari \\ Nagar, New Delhi-110 029, India
}

SUMmaRY The manifest (dry) refractions of 165 eyes of 86 patients, aged 6 to 75 years, were studied on the Nikon Auto Refractometer NR-1000F. The results obtained were compared with the clinical refractive data, and they were analysed for degree of agreement of various refractive components in different types and grades of refractive errors in the separate age groups. On the NR-1000F the spherical and cylindrical components and spherical equivalents skewed towards more minus (or less plus), especially so in emmetropes, low hypermetropes, and low myopes. This error declined with increasing age over 40 years and was also significantly lower in aphakia and mixed astigmatism. Determination of cylinder axis was found to be reliable on the NR-1000F. In spite of the obvious utility of the device its inbuilt automatic fogging system does not seem adequately to neutralise the patient's accommodative efforts, as the fixation target probably induces instrument myopia.

Automatic refractors have gained importance in recent years because of the busy clinical schedule of ophthalmologists and the increasing faith of patients in sophisticated mechanical devices. Many such refractometers, both subjective and objective, are now available, with steadily improving designs and claims to accuracy.' One of the latest objective models is the Nikon Auto Refractometer NR-1000F, which was recently installed in our centre. In view of several reports in the literature on the reliability and accuracy of various types of autorefractors ${ }^{2-8}$ we considered it worthwhile to undertake a systematic study to evaluate the NR-1000F Auto Refractometer (AR) before putting this instrument into routine general use in our busy hospital schedule.

\section{Materials and methods}

In the NR-1000F Auto Refractometer infrared rays are directed at the patient's fundus and the reflected light is detected by this instrument. A built-in microcomputer deduces the objective refraction in terms of sphere, cylinder, and axis, and then automatically displays this information, corrected for a 12 $\mathrm{mm}$ vertex distance. The AR also has the optional facility of automatic printout of the refractive data obtained, and is capable of recording a confidence

Correspondence to Dr Supriyo Ghose. value for each result to quantify numerically the reliability of the measurement. It completes each objective measurement in a total of 1.5 seconds, with the final measurement in only 0.5 seconds. The time taken for automatic fogging is 0.5 to 1 second with the patient seeing the fixation target, while the patient's accommodation is purportedly thoroughly relaxed by the inbuilt automatic fogging system. The machine can measure up to $\pm 15 \mathrm{D}$ sphere and $\pm 6 \mathrm{D}$ cylinder.

In accordance with the instructions in the operating manual supplied by the manufacturers the NR$1000 \mathrm{~F}$ is placed in a dimly lit, cool, and dust-free room. After a latent period of about 10 seconds on turning on the power switch a musical sound indicates that the machine is ready for use. The operator then manipulates the dioptre adjusting ring till the cross reticule at the centre of the viewfinder appears sharply in focus. The patient is seated comfortably, and the eye to be refracted is aligned to the correct level with the measuring head. A small child finds it easier to stand at the instrument. A model eye supplied may be used for operator practice as well as for an instrument check.

The green cross targets are aligned and centred by the operator with the peripheral red arrows moved out of the field of view to ensure precise positioning of the measuring head. The start button is depressed to activate the in-built automatic fogging system, and the refractive data are available within 1.5 seconds on 
Table 1 Refractive error and distribution by age of 86 patients (165 eyes)

\begin{tabular}{|c|c|c|c|c|c|c|c|}
\hline \multirow{2}{*}{$\begin{array}{l}\text { Age } \\
\text { group } \\
\text { (years) }\end{array}$} & \multirow{2}{*}{$\begin{array}{l}\text { No. of } \\
\text { patients }\end{array}$} & \multicolumn{5}{|c|}{ Type of refractive error (no. of eyes) } & \multirow{2}{*}{$\begin{array}{l}\text { Total } \\
\text { no. of } \\
\text { eyes }\end{array}$} \\
\hline & & Emmetropia & Myopia & $\begin{array}{l}\text { Hyper- } \\
\text { metropia }\end{array}$ & Aphakia & $\begin{array}{l}\text { Mixed } \\
\text { astig- } \\
\text { matism }\end{array}$ & \\
\hline$<10$ & 3 & 1 & 2 & 3 & 0 & 0 & 6 \\
\hline $11-20$ & 31 & 10 & 34 & 15 & 0 & 1 & 60 \\
\hline $21-30$ & 26 & 15 & 26 & 10 & 0 & 0 & 51 \\
\hline $31-40$ & 10 & 4 & 4 & 12 & 0 & 0 & 20 \\
\hline $41-50$ & 5 & 0 & 10 & 0 & 0 & 0 & 10 \\
\hline$>50$ & 11 & 0 & 3 & 7 & 6 & 2 & 18 \\
\hline Total & 86 & 30 & 79 & 47 & 6 & 3 & 165 \\
\hline
\end{tabular}

the digital display panel together with their confidence value. The confidence value tends to go down with improper alignment, eye movement, drooping eye lashes, small pupils, and opacities of the media. Further operating and maintenance information is detailed in the instruction manual.

A total of 172 eyes in 90 patients (eight were oneeyed) were tested in a comparative analysis. Their ages ranged from 6 years to 75 years (Table 1). Except for 19 patients who were medical or paramedical staff all the subjects in this study were those attending the outpatient department of our hospital for refractive problems.

Determination of the refractive error in all the eyes was done clinically as well as on the AR, but not necessarily by the same examiner. When both the tests were conducted by the same person, the clinical refraction was invariably done first so as to obviate any possible examiner bias in the clinical refractive data.

All refractions on the AR were performed without the use of mydriatics or cycloplegics. The clinical refractions were done either similarly (manifest or dry) or, if necessary, under the effect of cycloplegics (homatropine $2 \%$ drops or atropine $1 \%$ ointment) or mydriatics (phenylephrine 10\% drops), depending on the age of the patient, as is usual in clinical refractive practice. The final prescriptions were based on the objective clinical refraction data and the subjective acceptance by the patient, and the

Table 2 Mean differences and standard deviations between auto refractometer and clinical refractive data (165 eyes)

\begin{tabular}{lllll}
\hline & $\begin{array}{l}\text { Spherical } \\
\text { equivalent } \\
(D)\end{array}$ & $\begin{array}{l}\text { Cylinder } \\
\text { power } \\
(D)\end{array}$ & $\begin{array}{l}\text { Cylinder } \\
\text { axis } \\
\left({ }^{\circ}\right)\end{array}$ & $\begin{array}{l}\text { Aniso- } \\
\text { metropia* } \\
(D)\end{array}$ \\
\hline Mean & -0.58 & -0.31 & 6.43 & +0.13 \\
SD & \pm 0.79 & \pm 0.62 & \pm 9.85 & \pm 0.56 \\
\hline
\end{tabular}

*The + sign indicates more anisometropia on NR-1000F than shown by clinical refractive data. prescriptions were then compared with the AR printout data.

Results on the AR with a confidence value of less than 90 were rejected. A minimum of three readings were obtained for each eye tested, and the reading with the maximum confidence value in each eye was selected for comparison. In those few eyes with two or three readings with identical confidence values the average of each component (sphere, cylinder, and axis) was taken into account. In some eyes not even one reading with a confidence value over 90 could be attained: these were rejected for the purposes of comparison.

The comparative data obtained on the AR and by clinical refraction were analysed for degree of agreement for various types and grades of refractive error and by age groups.

\section{Results}

Of the total number of 172 eyes tested the data from seven had to be discarded for the purposes of the comparative study, as an AR reading with a confidence value of over 90 could not be obtained in spite of repeated examinations. Thus the clinical and AR data in 165 eyes of 86 patients were finally compared and analysed. Their refractive error and distribution by age are depicted in Table 1 . Of these 86 patients 47 were males and 39 females.

The mean difference and standard deviations (SD) in spherical equivalent data and cylindrical power and axis are depicted in Table 2. The comparative analysis for various types and grades of refractive error is given in Table 3 and the percentage agreement for different refractive components in Table 4.

The cylinder power data are shown in Tables 3 and 4 and the cylinder axis data in Tables 4 and 5. The variations of degree of agreement in the different age groups are recorded in Table 6.

When comparing the spherical equivalent data obtained clinically and by AR for the two eyes of the same patient we analysed the degree of aniso- 
Table 3 Degree of difference between the Auto Refractometer data and clinical refractive data for various refractive components in different types and grades of refractive error (165 eyes)

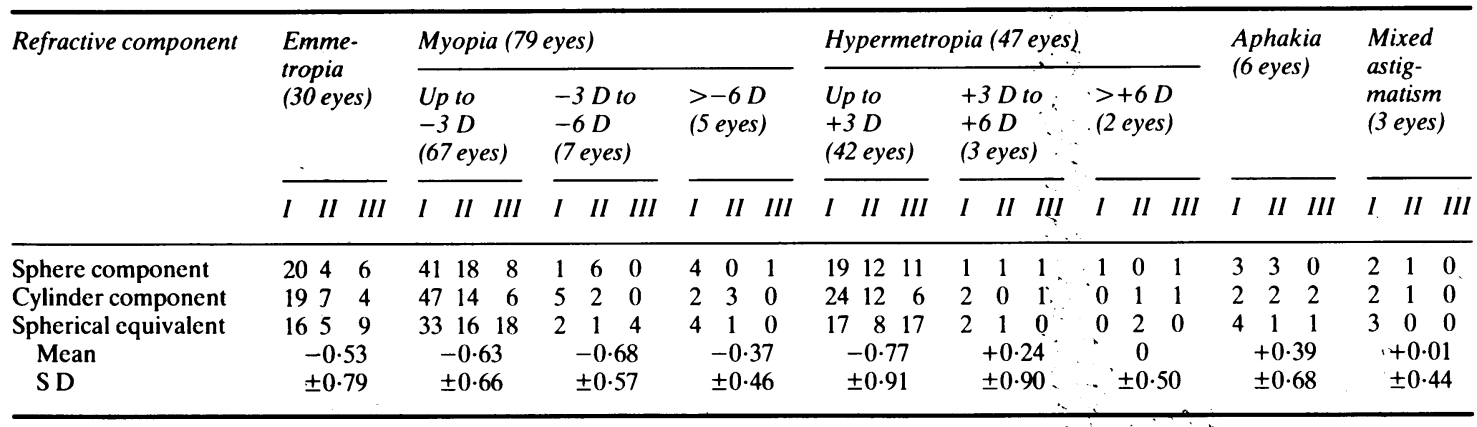

I: Difference less than or equal to 0.50 D.

II: Difference more than 0.50 D up to $1.00 \mathrm{D}$.

III: Difference more than 1.00 D.

Table 4 Percentage agreement between auto refractometer data and clinical data of various refractive components (165 eyes)

\begin{tabular}{|c|c|c|c|c|c|c|c|c|}
\hline \multirow{2}{*}{\multicolumn{2}{|c|}{$\begin{array}{l}\text { Spherical } \\
\text { equivalent } \\
\text { (D) } \\
\pm 0.50 \pm 1.00\end{array}$}} & \multirow{2}{*}{\multicolumn{2}{|c|}{$\begin{array}{l}\text { Sphere } \\
\text { component } \\
(D) \\
\pm 0.50 \pm 1.00\end{array}$}} & \multirow{2}{*}{\multicolumn{2}{|c|}{$\begin{array}{l}\text { Cylinder } \\
\text { component } \\
\text { (D) } \\
\pm 0.50 \pm 1.00\end{array}$}} & \multicolumn{3}{|l|}{ Cylinder axis } \\
\hline & & & & & & $\begin{array}{l}\text { Equal to or } \\
\text { under } \\
10^{\circ}\end{array}$ & $\begin{array}{l}\text { More than } \\
10^{\circ}\end{array}$ & $\begin{array}{l}\text { No cylinder } \\
\text { in one of } \\
\text { the testings }\end{array}$ \\
\hline $52 \%$ & $70 \%$ & $56 \%$ & $84 \%$ & $61 \%$ & $86 \%$ & $37 \%$ & $25 \%$ & $38 \%$ \\
\hline
\end{tabular}

Table 5 Degree of agreement of cylinder axis between auto refractometer and clinical refractive data in different types and grades of refractive error (165 eyes)

\begin{tabular}{|c|c|c|c|c|c|c|c|c|c|}
\hline & \multirow[t]{2}{*}{ Emmetropia } & \multicolumn{3}{|c|}{ Myopia } & \multicolumn{3}{|c|}{ Hypermetropia } & \multirow[t]{2}{*}{ Aphakia } & \multirow{2}{*}{$\begin{array}{l}\text { Mixed } \\
\text { astig- } \\
\text { matism }\end{array}$} \\
\hline & & $\begin{array}{l}\text { Up to } \\
-3 D\end{array}$ & $\begin{array}{l}-3 D \text { up to } \\
-6 D\end{array}$ & $\begin{array}{l}\text { Equal to or } \\
\text { more than } \\
-6 D\end{array}$ & $\begin{array}{l}\text { Up to } \\
3 D\end{array}$ & $\begin{array}{l}3 D \text { up to } \\
6 D\end{array}$ & $\begin{array}{l}\text { Equal to or } \\
\text { more than } \\
6 D\end{array}$ & & \\
\hline Cylinder axis within $10^{\circ}$ & 7 & 28 & 4 & 1 & 16 & 1 & 0 & 2 & 2 \\
\hline Cylinder axis difference $>10^{\circ}$ & 1 & 21 & 3 & 4 & 9 & 0 & 0 & 3 & 1 \\
\hline No cylinder in one of the testings & 22 & 18 & 0 & 0 & 17 & 2 & 2 & 1 & 0 \\
\hline Total ( 165 eyes) & 30 & 67 & 7 & 5 & 42 & 3. & 2 & 6 & 3 \\
\hline
\end{tabular}

Table 6 Degree of difference between auto refractometer data and clinical refractive data for various age groups in different types of refractive error ( 165 eyes)

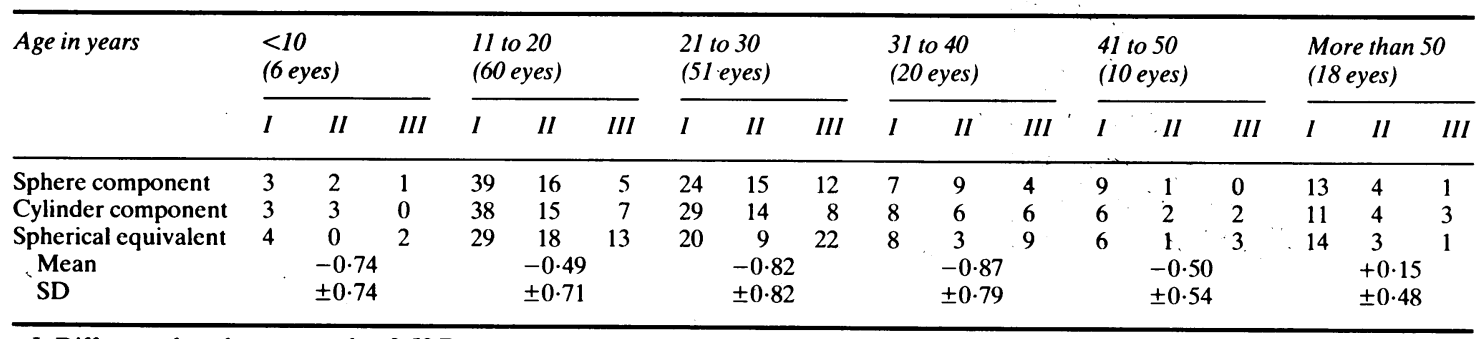

I: Difference less than or equal to $0.50 \mathrm{D}$.

II: Difference more than 0.50 D up to $1 \cdot 00 \mathrm{D}$.

III: Difference more than 1.00 D. 


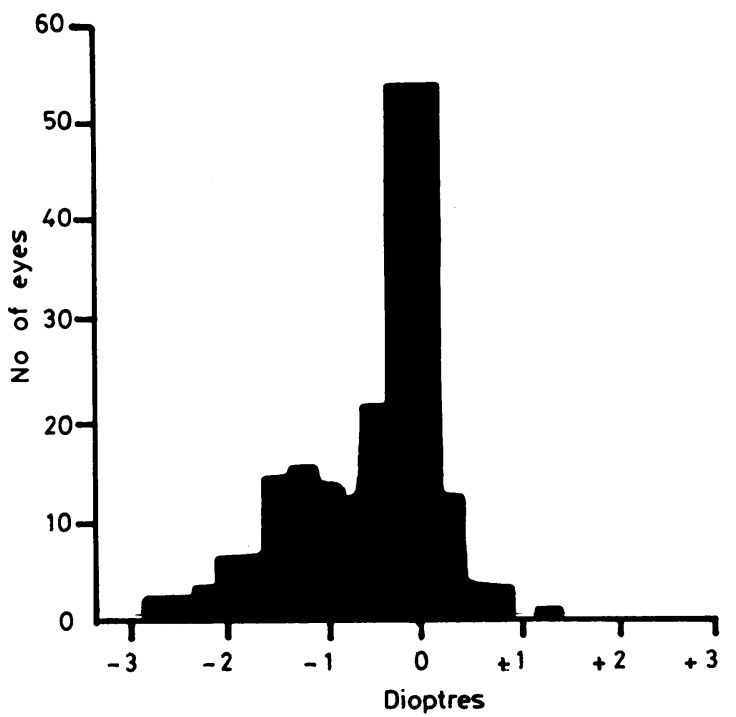

Fig. 1 Spherical equivalent values of 165 eyes: $a$ comparison between $N R-1000 F$ and clinical refractive data.

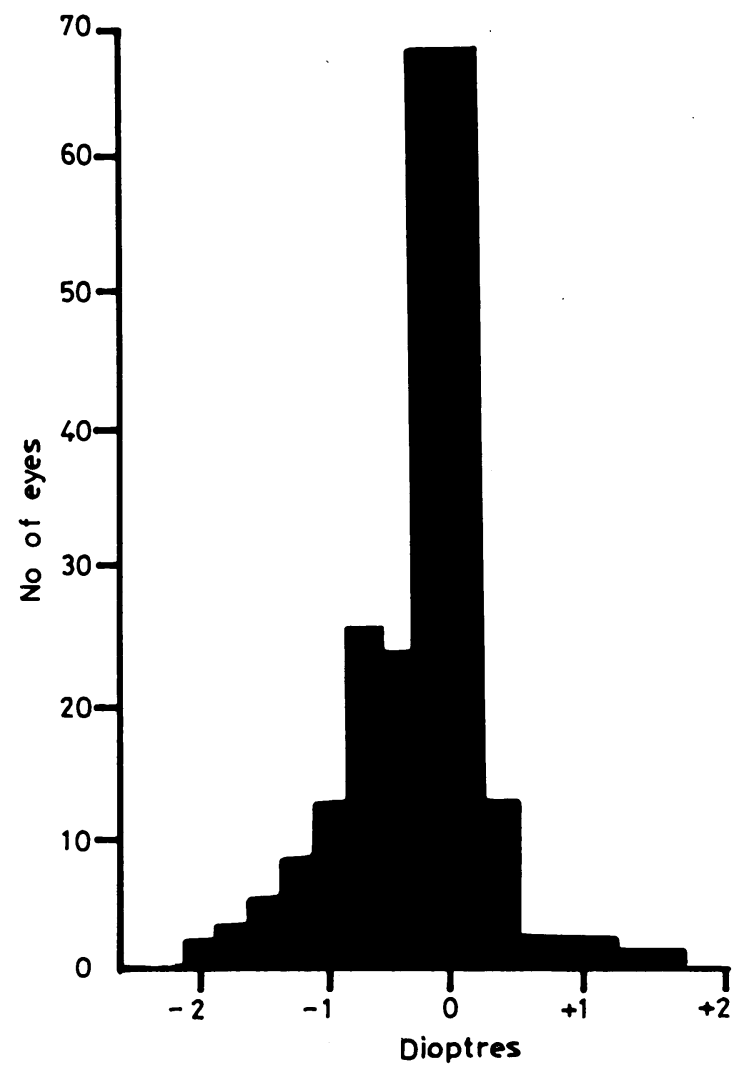

Fig. 3 Cylinder power data of 165 eyes: a comparison between NR-1000F and clinical refractive data.

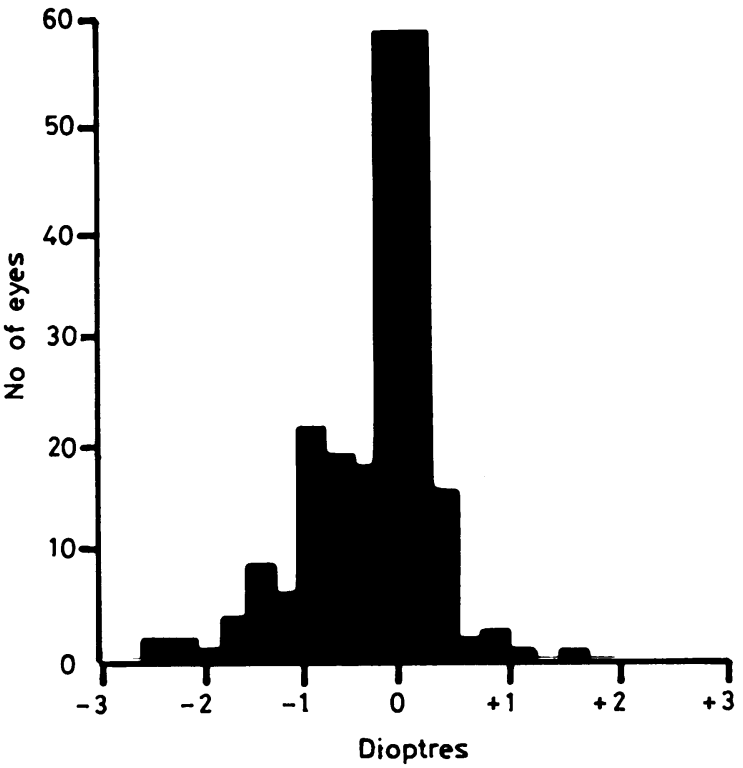

Fig. 2 Sphere component values of 165 eyes: a comparison between NR-1000F and clinical refractive data.

metropia by each method. It was found that the mean difference was $+0.13 \mathrm{D}(\mathrm{SD} \pm 0.56 \mathrm{D})$, the + sign here indicating a greater amount of anisometropia for AR data than clinical refractive data. Interestingly, a 'reversal' in anisometropia was noted in 10 of our 79 cases where both eyes were tested, that is, clinical data showed more plus for one eye and AR data more plus for the other eye.

\section{Discussion}

In our study eyes with a confidence value reading of less than 90 as indicated on the AR printout were rejected for the purposes of comparative analysis. Examiner bias was obviated by the methods detailed earlier.

It is evident from Figs. 1 and 2 and Table 2 that the $\mathrm{AR}$ recordings are, by and large, skewed strongly towards more minus or less plus than our clinical refractive data. It is also obvious from Table 3 that this skew deviation towards greater minus is more

In Figs. 1-3 the abscissa denotes the various degrees of dioptric difference observed between the AR and clinical refractive data, plotted against the corresponding number of eyes shown on the ordinate. The + sign in the abscissa signifies that the AR reading was more hypermetropic (or less myopic) than that of the clinical refraction, and the - sign indicates that the AR reading was more myopic (or less hypermetropic) than that of the clinical refraction. Note that the AR recordings skew towards more minus (or less plus) than our clinical refractive data. 
pronounced in the patients with emmetropia, low myopia (up to - 3 D), and low hypermetropia (up to +3 D). The minus error tends to come down in the higher myopic and hypermetropic groups, though the number of eyes in these groups is understandably small, as is only to be expected in a standard population sample. The reason for this difference seems obvious, namely, eyes with emmetropia or low refractive errors were capable of a fair degree of accommodation which the in-built automatic fogging system of the NR-1000F failed adequately to neutralise. The declining percentage of eyes showing higher minus error on comparison (more than $-1 \mathrm{D}$ ) with increasing age over 40 years (Table 6) also supports this view of incomplete accommodative neutralisation on AR, since the reduced capacity of accommodation with increasing age obviously tends to reduce this error. That the minus error in the few aphakic patients tested was significantly lower corroborates our explanation. For obvious reasons patients with higher refractive errors did not wish to accommodate significantly, and therefore the minus error recorded was much less in these eyes (Table 3).

It thus seems that an objective refractometer like NR-1000F would be far more accurate in aphakic patients and those with high refractive errors, but much less reliable in emmetropes and those with low refractive errors, especially in younger patients. The agreement of AR with clinical refractive data was very good in the three cases of mixed astigmatism tested (Tables 3 and 5); it is well known that such eyes hardly tend to accommodate so as to maintain the retinal image at or about the circle of least diffusion.

Variation in our results as compared with those of other studies may be partly attributable to basic differences in the methods of analysing the data. Our system of segregating eyes into graded refractive error groups and age groups seems to be a far more critical method of analysis than previous workers have used.

Evaluation of this type has been attempted to some extent earlier ${ }^{8}$ on a subjective refractor (SR III), but no comments are available on the distribution by age. Subjects below the age of 20 were not included in that analysis," and in another studys no information on the age of the patients was given. It has also been suggested ${ }^{x}$ that the inclusion of younger subjects would lower the extent of agreement between clinical and automatic refractions, and in order to evaluate the effect of overaccommodation on their SR III refractive data a study involving a large number of younger subjects would be needed. ${ }^{\text {. }}$ Our analysis has included 66 eyes of patients under 20 years of age, and the results of these have certainly borne out the suspicions harboured by previous workers. ${ }^{*}$
On clinical refractive assessment 123 out of 165 eyes $(74.5 \%)$ showed no cylindrical error, whereas only 24 eyes $(14.5 \%)$ showed purely spherical error on the AR. It seems that the cylindrical values obtained on the AR may often not be of clinical significance in many eyes.

The value of the cylindrical component obtained on the AR, like those for the spherical component and spherical equivalents, are also skewed towards more minus or less plus when compared with clinical refractive data (Fig. 3, Table 2). This observation has also been made earlier on the SR III subjective refractor. ${ }^{*}$ Again, like the spherical data, the skew deviation for cylinder power towards greater minus is more pronounced in emmetropic, low myopic, and low hypermetropic groups. The difference in agreement between the AR and clinical data on the cylindrical component in the various age groups (Table 6) was not significant. This is to be expected, as the cylindrical power in a given eye is unlikely to be influenced by accommodative effort, in contrast to the spherical component.

The mean difference of cylinder axis values determined clinically and on the AR was $6.43^{\circ}$ (Table 2) with a standard deviation of $\pm 9 \cdot 85^{\circ}$. The determination of axis by the AR appears to be quite reliable and accurate. Similar observations have been made before ${ }^{8}$ though on a subjective refractor axis readings were found to vary in the same eye on separate testings. The reliability of the NR-1000F for cylinder axis determination seems to be greater than its accuracy in recording the spherical and cylindrical powers.

Our comments on the anisometropic data and 'reversal' in anisometropia are in fair agreement with the earlier observations by Perrigin et al. ${ }^{.}$These should be kept in mind while clinically prescribing on data obtained by automatic refractors, subjective or objective.

In seven of the total of 172 eyes in our study the recommended confidence value of over 90 could not be obtained despite repeated efforts by the patient as well as the examiner, without there being any apparent reason. Conversely, a higher confidence value rating on the AR did not always prove more reliable than the figures obtained with a lower confidence value, when compared with the clinical refractive data for that eye. The reason for this is not clear. The operating manual supplied with the NR$1000 \mathrm{~F}$ also comments on this apparent paradox, though no explanation is suggested.

It must be pointed out that the $\mathrm{AR}$ has proved to be extremely useful in the odd eye where retinoscopy and clinical improvement proved most unsatisfactory, especially in those eyes with corneal opacity.

We suggest that automatic refractometers like the 
NR-1000F have a place in busy ophthalmic clinics, such as in a large hospital, and should be handled by trained operators to give baseline refractive data. The AR reading should not be prescribed straight away without carefully checking the acceptance subjectively, or using it as a guideline in case the clinical refraction presents difficulties. Automatic refractors are doubtless an invaluable aid to the specialist, but they do not and should not replace the art of clinical refraction.

Inadequate neutralisation of the patient's accommodative efforts by the in-built automatic fogging system of the NR-1000F appears to be the major drawback of this objective autorefractometer. It is possible that in spite of the fogging system the fixation target still induces a significant degree of instrument myopia. Further work is being carried out on the AR with eyes under the effect of cycloplegics.

\section{References}

1 Guyton DL. Aụtomated refraction. Invest Ophthalmol Vis Sci 1974; 13: 814-8.

2 Drance SM, Mitchell DWA, Schulzer M. Studies of an automatic refraction machine. Can J Ophthalmol 1975; 10: 462-8.

3 Polse KA, Henton EK. An automatic objective optometer. Arch Ophthalmol 1975; 93: 225-31.

4 Dyson C. A clinical study of the autorefractor, an automatic refracting device. Can J Ophthalmol 1977; 12: 29-33.

5 Bannon RE. A new automated subjective optometer. Am J Optom Physiol Opt 1977; 54: 433-8.

6 Pappas CJ, Anderson DR, Briese FW. Clinical evaluation of the 6600 autorefractor. Arch Ophthalmol 1978; 96: 993-6.

7 Pappas CJ, Anderson DR, Briese FW. Is the autorefractor reading closest to manifest refractions? A comparison of the patient's previous spectacles and the 6600 autorefractor reading. Arch Ophthalmol 1978; 96: 997-8.

8 Perrigin DM, Perrigin J, Grosvenor T. A clinical evaluation of the American Optical SR III subjective refractor. Am J Optom Physiol Opt 1981; 58: 581-9.

Accepted for publication 8 July 1985. 